\title{
RESERCH OF POSSIBILITY OF INCREASING EFFICIENCY OF VORTEX CAVITATOR BY RESONATOR DESIGN IMPROVEMENT
}

\author{
Nikita Kokorin ${ }^{1}$, Andrey Gordeev ${ }^{1}$, Andrey Brysin ${ }^{2}$ \\ ${ }^{1}$ Nizhny Novgorod State Agricultural Academy, Russia; \\ ${ }^{2}$ Russian Technological University, Russia \\ nikia_6500@mail.ru,g0rd.ab@mail.ru,brysin@rambler.ru
}

\begin{abstract}
The work of the vortex cavitator is based on the phenomenon of acoustic cavitation. Due to the highspeed collapse of steam-air cavities (cavitation cavities), a significant amount of useful energy is released in the local volume of the liquid. This fact can be used, for example, for highly efficient heating, dispersion with simultaneous disinfection of peat, manure, for presowing treatment of seeds and the implementation of many other processes. The resonator of the vortex cavitator plays a significant part in the amount of energy realized in the cavitation process. In the resonator body, the acoustic signal is amplified by the formation of a standing wave (the amplitude and q-factor increase). It was established that the reflection of the acoustic wave in the resonator body is not absolute due to the difference between the direct and reflected sound waves. The formation of a standing wave is prevented by a different degree of reflection from the structural elements adjacent to the body, which have different mass, stiffness and methods of attachment. This asymmetry of the resonator body at the points, where it is connected to the cyclone and the return pipeline, reduces the efficiency of the installation workflow. For the cavitator efficiency upgrading (increasing the amplitude of sound vibrations and the q-factor of the oscillating circuit), it is proposed to replace the existing resonator of the device with an improved one, with optimal geometric parameters for a more favorable behavior of the cavitation process. This event will allow: to increase the level of released energy due to the increased value of the acoustic signal amplitude; to reduce the consumption of energy input, with a constant output power of the installation, due to the reduced level of attenuation of sound waves (increase the q-factor) in the resonator body.
\end{abstract}

Keywords: acoustics, cavitation, resonator, frequency response, pipes, water.

\section{Introduction}

At the Engineering Faculty of the Nizhny Novgorod State Agricultural Academy a number of vortex and hydrodynamic cavitators were created [1] (Fig. 1), the application of which speeds up and qualitatively improves technical processes (mixing, dispersion, coagulation, disinfection, etc.) (Fig. 2). Also of interest is the effect of acoustic cavitation on biological objects [2]: rapid germination of grain by treating it in the operating space of the device, pre-treatment of seed material, giving an increase to a high germination and vigor of crops, accelerated development of plants when using water treated in cavitator [3] and others.

a)

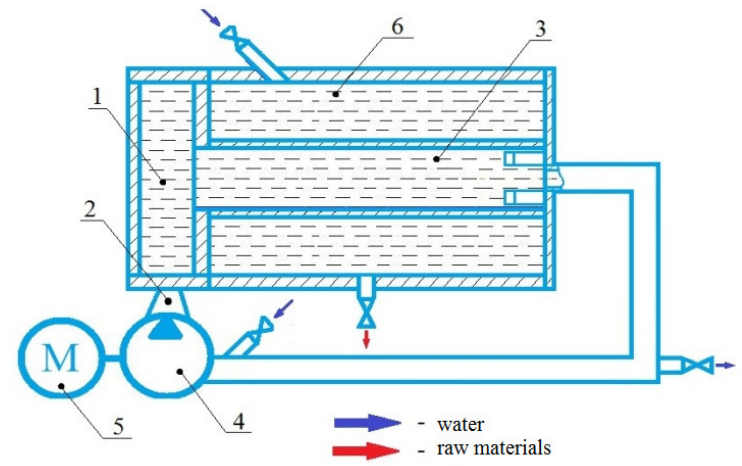

b)

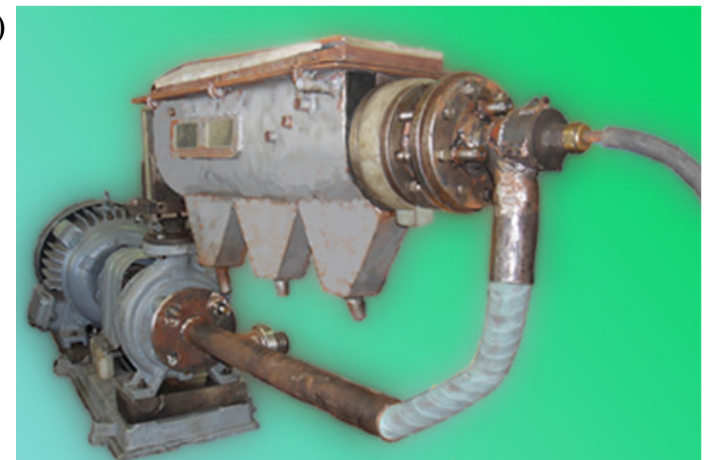

Fig. 1. Scheme of acoustic cavitation installation with a water-jacket (a) and its appearance (b): 1 - scroll cavitator (the vortex chamber); 2 - tangential nozzle; 3 - resonator; 4 - hydraulic pump; 5 - engine; 6 - water-jacket

Acoustic cavitation characterized by high efficiency of work performed, but the process of converting hydraulic power into sound is not perfect. The transition percent of input of energy in acoustic form does not exceed 10 [4]. This circumstance is sending to the search for measures to improve the efficiency of this conversion. 


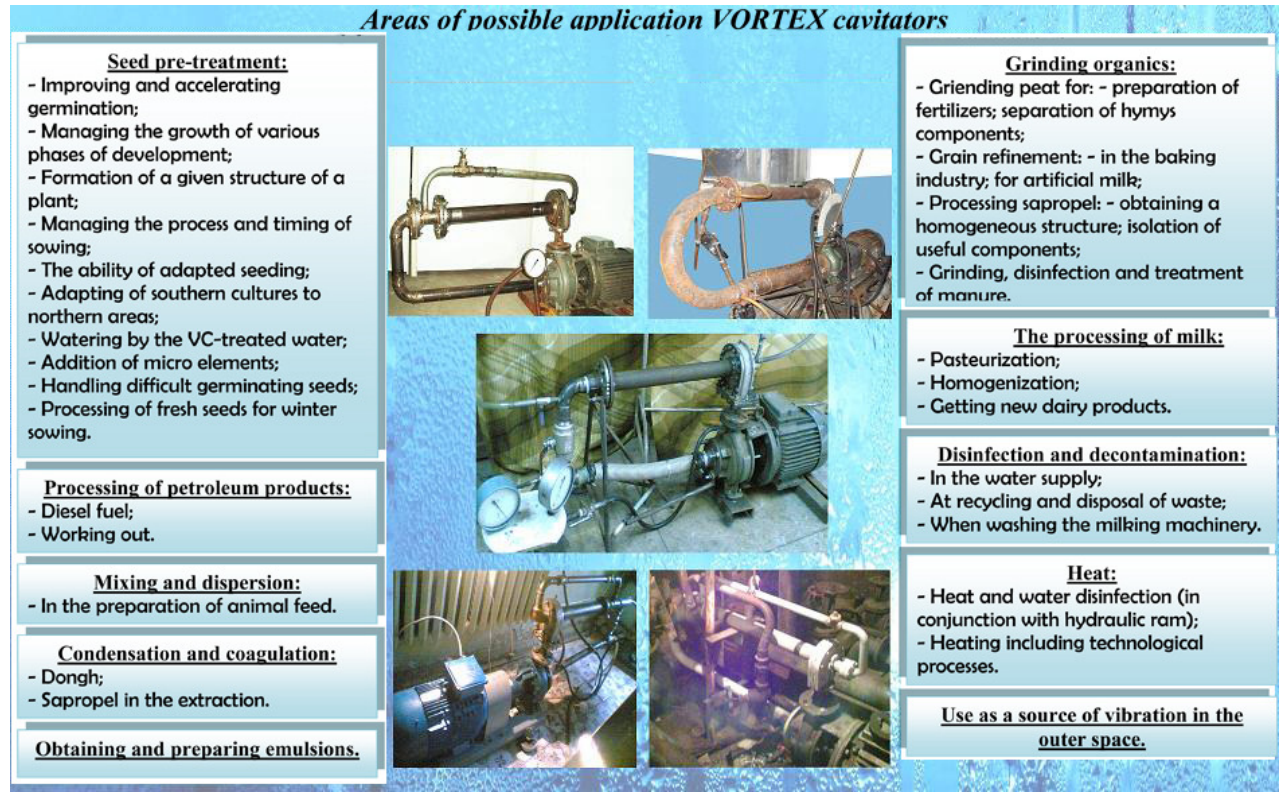

Fig. 2. Cavitation-acoustic technology

One of the methods for solving the problem of thermoacoustic energy conversion in a resonator is the use of thermoacoustic power amplifiers. An example of such an amplifier is a differentially heated regenerator installed near the velocity node of the resonator [5]. However, this method involves a significant complication of the device design and additional energy consumption for its workflow.

The most straightforward way of reducing the unnecessary cost of power is creating the right (that is, the required parameters: amplitude, intensity, frequency) acoustic field inside the working volume and reducing the loss of sound energy.

\section{Materials and methods}

The resonator provides most of the energy in the cavitation process, due to the formation of a standing wave of the acoustic signal, created by the vortex chamber (the amplitude and quality factor of sound vibrations increases).

The quality factor is a measure of the relative dissipation of energy, that is, the ratio of the energy stored in the oscillatory system to the energy lost by the system in one period of oscillation [6;7]. Its value is inversely proportional to the damping decrement:

$$
Q=\frac{1}{D}=\frac{\omega_{0}}{2 \cdot \alpha},
$$

where $D$-damping rate of the oscillations of the system;

$\omega_{0}$ - natural frequency of the system; $\mathrm{Hz}$;

$\alpha-$ attenuation coefficient.

The sound signal processing, when determining the quality factor, was carried out using the SpectraPLUS 5.0 software (Fig. 3), which is necessary to calculate the relaxation time of the pipe sound vibrations $(\tau)$, and then the attenuation coefficient $(2)$ :

$$
\alpha=\frac{1}{\tau},
$$

where $\tau$-relaxation time (the damping of sound vibrations) of oscillating circuit; sec.

The measurements of the frequency response of the resonators were carried out with triplicate. 


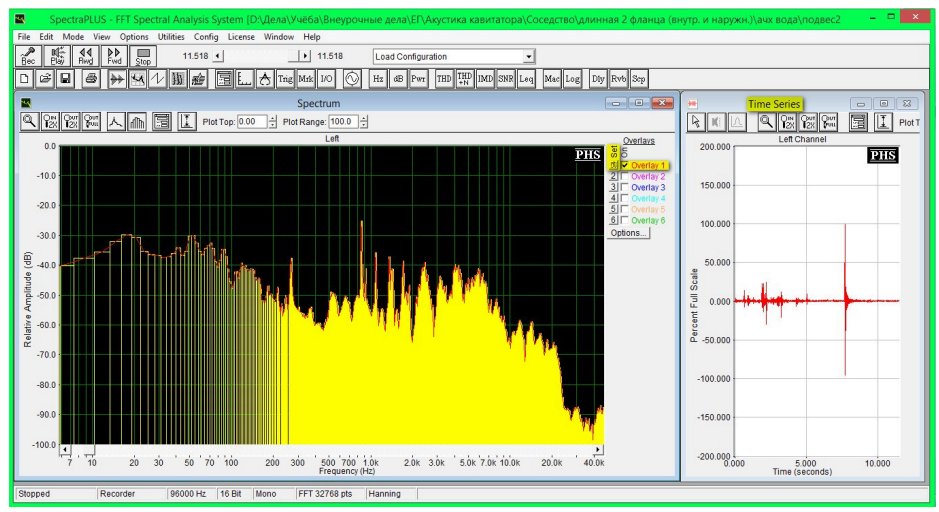

Fig. 3. SpectraPLUS 5.0 program window

\section{Results and discussion}

A negative feature of the workflow is that it contains the additional sources of vibration: engine, pump and also single elements of the construction. These objects emit elastic vibrations of different frequencies and amplitudes. In the end, on the generated waves are superimposed external disturbances, reducing the efficiency of acoustic cavitation, as a result, except the useful range (1$3 \mathrm{kHz}$ ) generated by a wide range of frequencies spending additional energy (Fig. 4) [8].

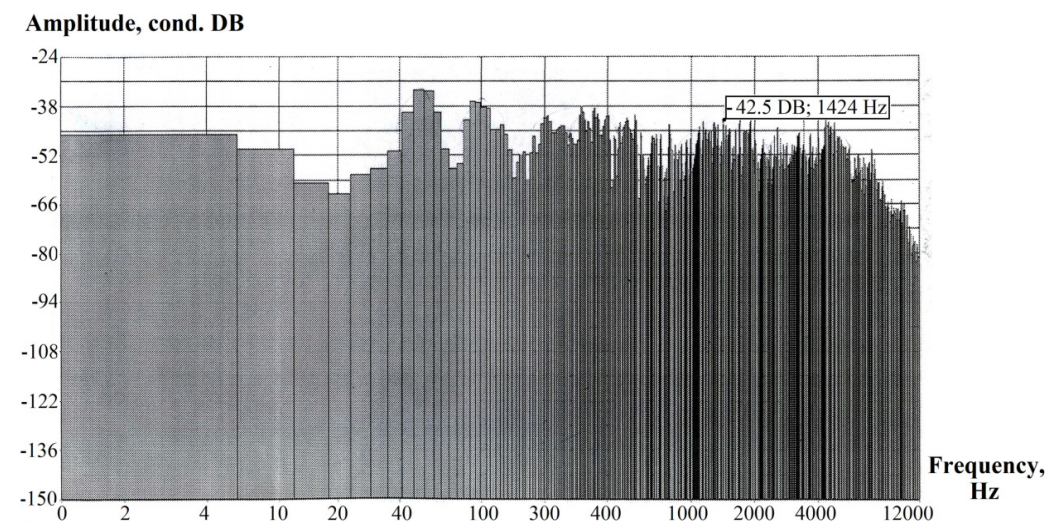

Fig. 4. Frequency response sound vibrations generated by the installation

On the other hand, the elastic waves, which are specially produced in the vortex chamber, should be in the body of the resonator on its ending reflect not absolute but a loss, because this part of the elastic energy is distributed to the adjacent metal structures due to their direct contact [9]. As a result, the amplitude of elastic vibrations produced becomes much lower than the expected values, and the values of their frequencies are in a state of drift, because the process of reflection takes the probabilistic nature. In this case, the input energy is dispersed in hard links in contact with the body and adjacent structures.

These negative effects can be mitigated to an extent by installing the rubber damping spacers (bushings) between the adjacent structural elements (Fig. 5).

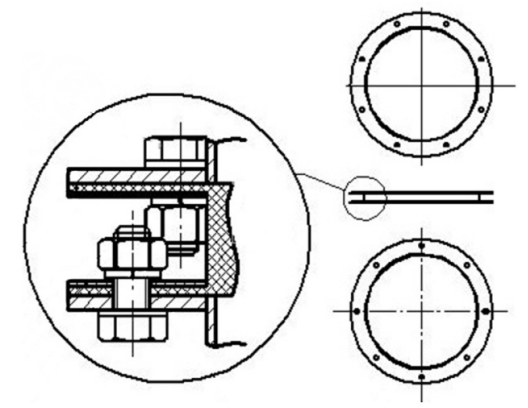

Fig. 5. Vibration-absorbing rubber insert 
This fact is confirmed by the data obtained in the experiment on the measurement of amplitudefrequency characteristics (hereinafter AFC) of the resonator in three states: no contact with adjacent elements (on the suspension), the contact of the resonator with a solid surface and the break hard contact with the rubber strips (Table 1).

Table 1

Results of the AFC

\begin{tabular}{|c|c|c|c|c|c|c|}
\hline \multirow{2}{*}{ No. of frequency } & \multicolumn{6}{|c|}{ Frequency $(\boldsymbol{f} ; \mathbf{H z})$ and amplitude $(\boldsymbol{A}$; cond. DB)* } \\
\cline { 2 - 7 } & \multicolumn{2}{|c|}{ Floor } & \multicolumn{2}{c|}{ Suspension } & \multicolumn{2}{c|}{ Rubber } \\
\cline { 2 - 7 } & $\boldsymbol{f}$ & $\boldsymbol{A}$ & $\boldsymbol{f}$ & $\boldsymbol{A}$ & $\boldsymbol{f}$ & $\boldsymbol{A}$ \\
\hline 1 & 838.1 & -33.0 & 838.0 & -23.2 & 838.1 & -19.8 \\
\hline 2 & 1060.8 & -42.4 & 1060.6 & -33.7 & 1072.3 & -19.6 \\
\hline
\end{tabular}

* - conditional value (scale of amplitudes is not calibrated in decibels - specific software package SpectraPLUS 5.0)

From the analysis of the results of the frequency response, it follows that at the same frequency of sound waves, and the first and second mode, the amplitude value of the signal maximum for the latter case, that is, when used as damping elements, rubber seals (Fig. 6, 7).

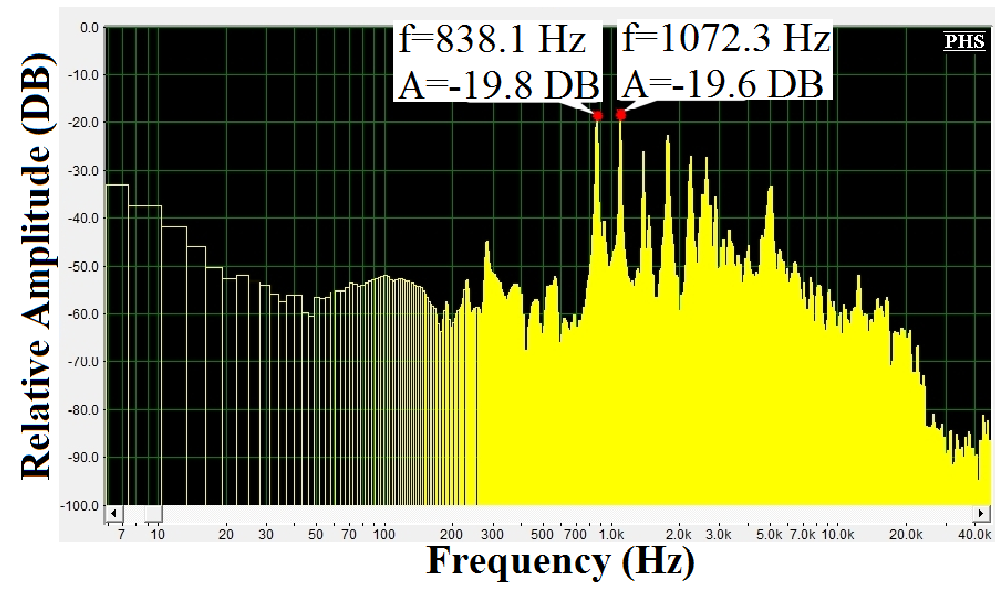

Fig. 6. AFC of a pipe resonator in contact with the rubber gasket

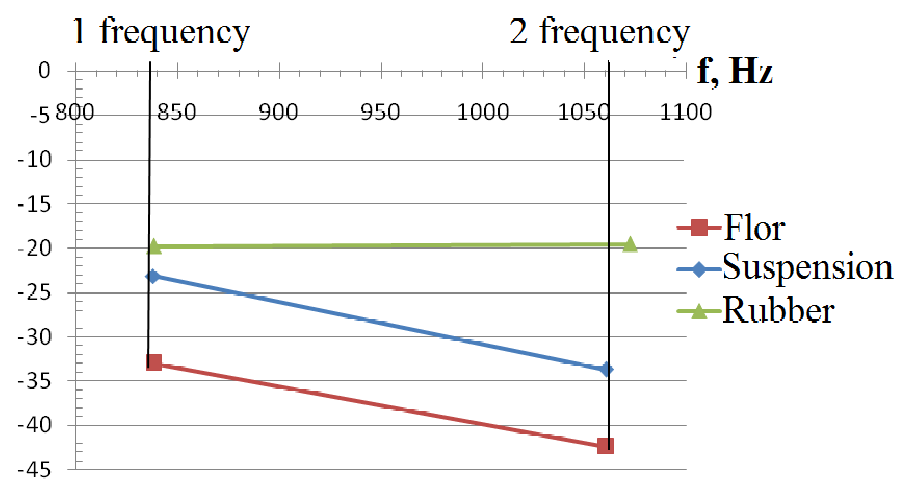

A, cond. DB

Fig. 7. Amplitude of the acoustic oscillations of the resonator type in contact with its material

A similar question was considered in the study on acoustic performance of a water muffler for the gear pump [10]. Simulation results and experimental results show that the rubber tube with a compliant wall substantially contributes to the reduction of the noise generated by the gear pump, where there are acoustic noises, too.

Thus, in the installation of vibration-absorbing bushings in months-tick the connection of the resonator with the pump and return pipe allows to isolate the resonator housing from the frame of the 
oscillating system that eliminates the transmission, exposure to vibration from power plants, as well as care of the useful signal in the frame part.

The asymmetric conditions of the neighborhood of the resonator in the places of its connection to the cochlea and the return pipeline also significantly affect the characteristics of the produced standing wave. Moreover, the standing wave may not take place, because the different degree of reflection from adjacent the housing of structural elements having a different mass, stiffness, method of fixation, causes differences between the direct and reflected waves [11]. That is, between ways of fixing the housing on its length, diameter, wall thickness, its material properties and operating fluid should meet the following condition for the formation and subsequent maintenance of the standing wave.

During the experiments, were investigated in resonators:

- with different conditions of reflection - measuring the resonator (material - steel) by increasing the mass of flanges (Fig. 8), changing fluid density;
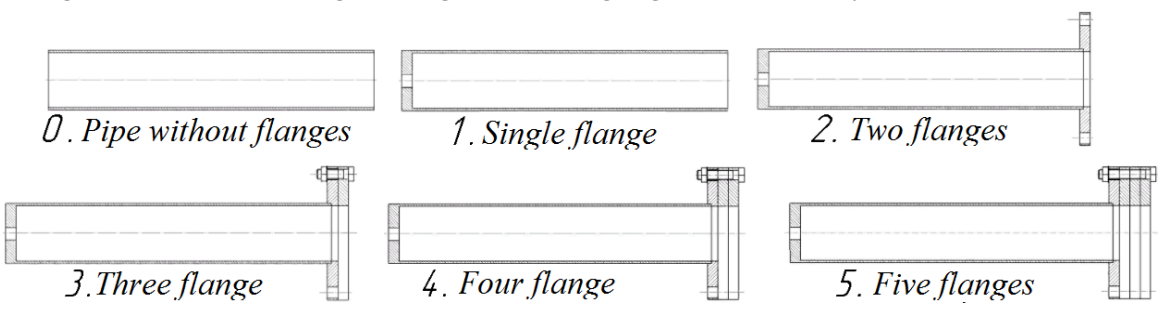

Fig. 8. Test samples of resonators

- made of different material (steel, cast iron, stainless steel, aluminum, copper);

- different geometry design (material - steel) (Fig. 9)

a)

b)
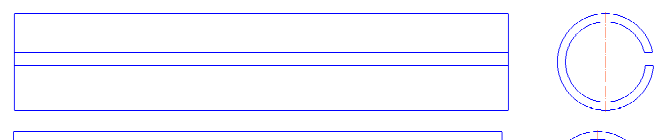

c)

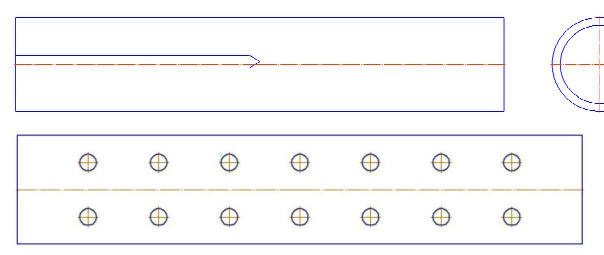

d)

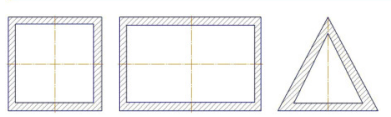

Fig. 9. Performance resonator: a - with a longitudinal slit along the entire length of the pipe;

$\mathrm{b}$ - with a longitudinal slit to $1 / 2$ the length of the pipe; $\mathrm{c}$ - with perforation;

$\mathrm{d}$ - different cross-sections, etc.

On these characteristics experimentally are set the following patterns:

1. The natural frequency of the resonator varies depending on the length of the pipe, diameter, wall thickness and density of the fill to the working environment, but it does not depend on the size and design of the neighbouring subassemblies - flange (Fig. 10) (in the figure indicated as "Mass with flanges").

a) Frequency, $\mathbf{H z}$ The first frequency

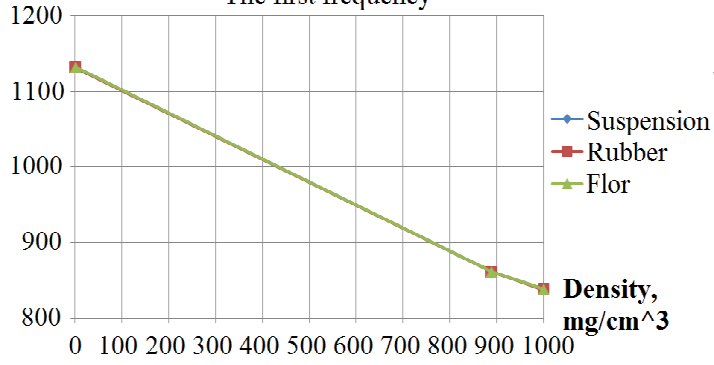

b)

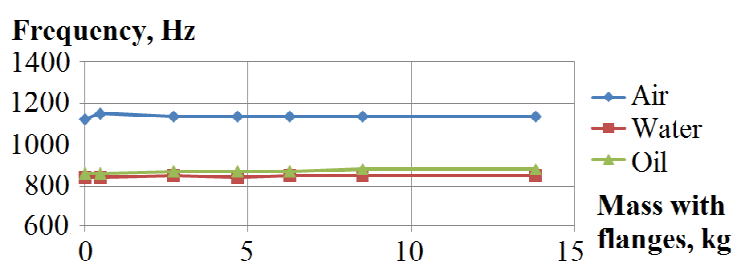

Fig. 10. Dependence of the frequency vibration of the resonator: a - density of the medium that fills interior space; $b$ - mass attached to the pipe flanges 
Thus, the acoustic characteristics of the resonator with the air are much above similar characteristics of oil and water.

2. The magnitude of the amplitude is influenced by:

- first, the conditions of reflection of the sound wave to the sample tube resonator without flanges (Fig. 11) (position 0) and with one inner flange (position 1), the amplitude minimum, and the sample No 2 with 2 flanges, as best reflected signal, respectively, the maximum amplitude;

- secondly, the asymmetry of the boundary conditions: with the increase added to one of the flanges of the mass, there is a tendency to decrease the amplitude.

However, during the filling of the resonator with a liquid (water, oil), the increase in the mass of the flanges, even when increasing asymmetry of the system (pipe resonator with flanges (Fig. 8)), provides the most reflect amounts ability.

3. At factor of quality, i.e. the measure of the relative energy dissipation of the sound wave, there is a similar situation: the minimal of this quantity for a bad reflection conditions (Fig. 12) (pipe No. 0, 1) and the suboptimal mass (No. 4 and then with increasing mass).

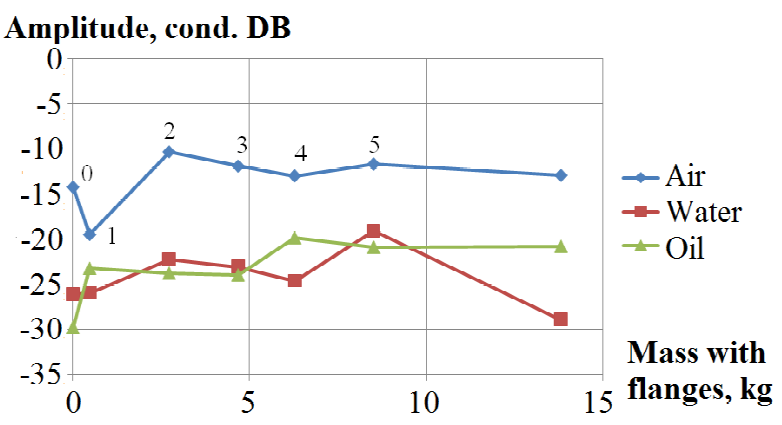

Fig. 11. Amplitude of the sound vibrations of the resonator from add to its flange mass

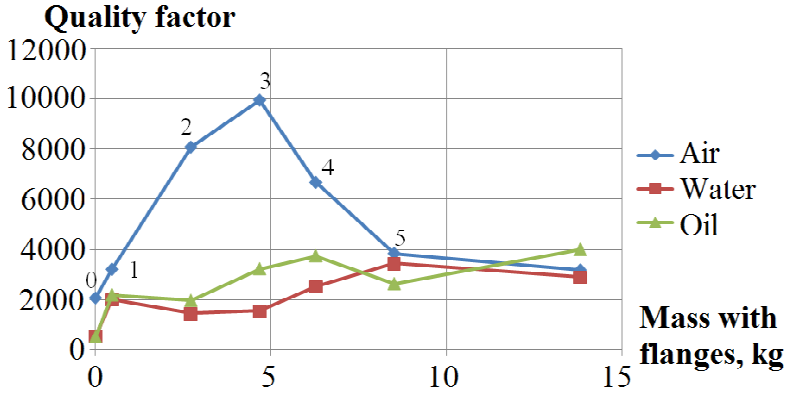

Fig. 12. Dependence of the quality factor sound vibrations of the resonator from add to its flange mass

Another important factor is the vibration quality of the case material, that is, its clinging properties. So, when manufacturing the body of material with viscous properties (e.g. lead), it would have a greater amount of decrement of attenuation, that is, the elastic force, when the oscillations are largely suppressed by the internal and external friction. Therefore, in this case, if any, we will not have a standing wave or for its maintenance we will need to spend a significant amount of energy.

In the manufacture of the housing from a material with ringing properties (e.g. bronze) it will have a smaller value of the damping factor (a big q-factor), therefore, to maintain the standing wave there needs to be less amount of input energy, either at the same level, the amplitude will be of great value. Similar processes take place in the bell in the Navy, Church bells, etc. [12].

From the analyzed variants of the resonator material: steel, stainless steel, aluminium, copper, cast iron, the best option to get a more powerful acoustic signal is a resonator made from steel (Table 2).

Table 2

\section{Results of the measurements of the quality factor $(Q)$ resonators made of different materials}

\begin{tabular}{|l|l|}
\hline \multicolumn{1}{|c|}{ Material } & \multicolumn{1}{c|}{$\mathbf{Q}$} \\
\hline Steel & 1467.9 \\
\hline Stainless steel & 1371.9 \\
\hline Aluminium & 1259.1 \\
\hline Copper & 1113.7 \\
\hline Cast iron & 563.2 \\
\hline
\end{tabular}

In addition, the material production has a significant effect on the AFC of the resonator given by its geometrical parameters. This fact is confirmed by the results of an experiment comparing acoustic 
characteristics of the buildings of the resonator with a longitudinal slit, with $1 / 2$ of the slit and without slit (Fig. 9) (Table 3).

Table 3

Results of the frequency response steel resonators various geometric performance

\begin{tabular}{|l|c|c|c|c|}
\hline \multicolumn{1}{|c|}{ Body of resonator } & $\boldsymbol{\omega 0}$ & $\mathbf{A 1} *$ & $\boldsymbol{\tau}$ & \multicolumn{1}{c|}{$\mathbf{Q}$} \\
\hline Steel - longitudinal slit & 644.0 & -34.2 & 7.4 & 2373.7 \\
\hline Steel - without an slit & 843.3 & -13.6 & 4.0 & 1698.7 \\
\hline Steel - 1/2 slit & 299.4 & -28.8 & 4.8 & 719.2 \\
\hline
\end{tabular}

* A1 - the amplitude of the first frequency; cond. DB

From the analysis of the data shown in the table, it follows that the magnitude of the frequency and amplitude of the acoustic signal the best example is the one-piece body of the resonator (without slit) with maximum settings: $843 \mathrm{~Hz}$. and -13.6 cond. DB. However, the most important parameter for reducing the energy intensity of the resonator, and in the general device it is the value of the q-factor of the oscillating system, so, than its value is bigger, then less the energy loss in the system over one oscillation (formula 1). [6]

Evaluating this parameter $(\mathrm{Q})$ revealed that the best acoustic characteristics of a resonator with a complete longitudinal slit having the maximum value of $q-2347$ units, but the smallest value of the amplitude - -34.2 cond. DB.

Thus, the quality of the oscillating system cannot be assessed only by the values of the frequency $\left(\omega_{0}\right)$ and amplitude (A). An important parameter is the value of the quality factor $(\mathrm{Q})$, which is as many times more, how much more the decay time of the sound vibrations of the system ( $\tau$ ) (Fig. 13).

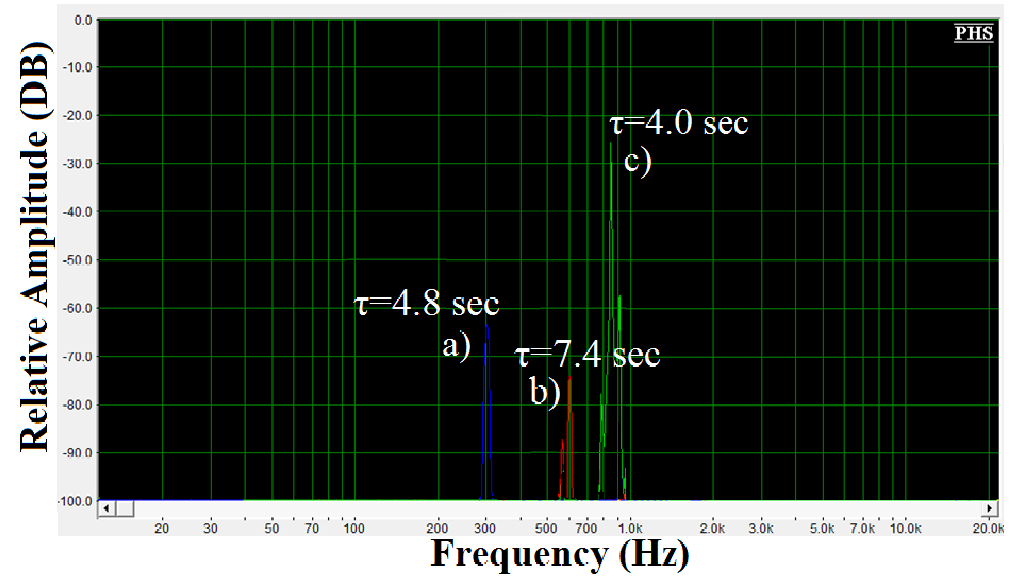

Fig. 13. Damped harmonic first mode of resonators: $a-1 / 2$ of the pipe slit;

$\mathrm{b}$ - with full longitudinal slit; $\mathrm{c}$ - without slit

This circumstance opens a wide range of possible options for structural changes of the resonator of the prototype devices having improved amplitude-frequency characteristics and reduced power consumption. The application of the best of these options will significantly reduce energy and resource consumption at constant quality with the help of the cavitator works.

\section{Conclusions}

1. It is appropriate to separate the resonator from the cavitator vibration eliminator.

2. The resonator should be performed in a tube with flanges, the amplitude and q-factor increase.

3. The mass of the flange and the ratio of their masses determine the maximum value of the amplitude and quality factor.

4. The fluid dynamic characteristics of the resonator are very different from the characteristics of the air frequency, amplitude and q-factor fall.

5. The implementation of the resonator of materials having "ringing" properties, opens up a whole direction of development of the device - search and creation of new ringing materials in the water for the manufacture of shells. 


\section{References}

[1] Ivanov E.G., Denisyuk E.A., Nosova I.A. etc. The taxonomy of vortex heat generators. Collection of scientific works NNSAA "Improvement of technical and operational processes and energy resources in agriculture", 2007, N. Novgorod, Russia, pp. 176-188.

[2] Ivanov E.G., Sinitsyn V.P., Tyutin V.V. Intensification of processes in heterogeneous media using the cavitation phenomena. Collection of scientific works NNSAA "Improvement of technical and operational processes and energy resources in agriculture", 2007, N. Novgorod, Russia, pp. 208215.

[3] Ivanov E.G., Kokorin N.V. Manifestation of information properties of water suffered cavitation in aftereffect via examples "memories of soils". Proceedings of $18^{\text {th }}$ International Scientific Conference "Engineering for rural development", May 22-24, 2019, Jelgava, Latvia, pp. 493-500.

[4] Timoshenko S.P. Oscillations in engineering. Moscow: Science, 1967. 444 p.

[5] Biwa T., Tashiro Y., Mizutani U. etc. Experimental demonstration of thermoacoustic energy conversion in a resonator. Physical Review E - Statistical, Nonlinear, and Soft Matter Physics, vol. 69, No 6, 2004.

[6] Savelev I.V. General Physics Course. Mechanics, vibrations and waves, molecular physics. First edition. Moscow: Science, 1970. 510 p.

[7] Kulikov G.V., Zung N.V. Influence of synchronization errors on the interference of M-FM signal receiving. Russian technological journal, vol. 7, No 5, 2019, pp. 47-61.

[8] $2609553 \mathrm{EN}$, F24J 3/00 invention patent "Device for heating fluid".

[9] Boguslavskii Iu.Ia. On the absorption and dispersion of sound waves in a two-phase medium. Acoustic Journal, vol. 24, No 1, 1978, pp. 46-52.

[10] Du T., Chu N., Cao L. etc. Study on acoustic performance of a water muffler for gear pump. International Journal of Acoustics and Vibrations, vol. 24, No 1, 2019, pp. 34-43.

[11] Likhopersky V.I. Beat periodic oscillations in the destruction of the vortex layers. Proceedings of the USSR Academy of Sciences. Fluid mechanics, No 2, 1977, pp. 160-162.

[12] Ivanov E.G. Evolution of hydrodynamic cavitator. Mechanization in Agriculture, vol. 2, 2015, pp. 17-21. 